\author{
P. M. Honoré \\ R. Jacobs \\ W. Boer \\ O. Joannes-Boyau
}

\section{Sepsis and AKI: more complex than just a simple question of chicken and egg}

Received: 9 November 2010

Accepted: 10 November 2010

Published online: 9 December 2010

(C) Copyright jointly held by Springer and ESICM 2010

This editorial refers to the article available at: doi:10.1007/s00134-010-2089-9.

P. M. Honoré $(\bowtie) \cdot$ R. Jacobs

ICU Department, Universitair Ziekenhuis Brussel,

Vrije Universitieit Brussel (VUB), 101, Laarbeeklaan,

1090 Jette, Belgium

e-mail: Patrick.Honore@uzbrussel.be; Pathonor@skynet.be

Tel.: +32-2-4749097

Fax: +32-2-4765179

W. Boer

Department of Anaesthesiology and Critical Care Medicine, Ziekenhuis Oost-Limburg, Genk, Belgium

O. Joannes-Boyau

Haut Leveque University Hospital of Bordeaux,

University of Bordeaux 2, Pessac, France

Acute kidney injury (AKI) in critically ill patients has been largely regarded as a consequence but not as a cause of sepsis $[1,2]$. Indeed, most studies focused on incidence, risk factors, and prognosis of sepsis-induced AKI [3] demonstrating its association with a high mortality rate [4] as well as longer hospital stay [5]. Studies addressing the underlying histopathology and pathophysiology of septic AKI have revealed that renal blood flow, both medullar and cortical, was maintained and even increased during severe septic shock [6], undermining earlier concepts and reinforcing the hypothesis that septic AKI is a separate physiological entity from nonseptic
AKI. Further evidence for this was provided when it was demonstrated that there was an important role for apoptosis, rather than just necrosis, in sepsis and septic shock $[7,8]$.

In this issue of Intensive Care Medicine, Mehta et al. [9] describe a multicenter, observational study, looking at the relationship between AKI and sepsis. Undoubtedly the major finding was that more than half of patients defined as nonseptic during presentation with AKI developed sepsis during hospitalization, and $50 \%$ of them within 5 days after AKI diagnosis. Of course, one of the strengths of the study is its multicenter set-up limiting bias related to factors associated with any particular single center. However, there are limitations to the study: first, patients were included upon nephrology consultation and therefore both delay in diagnosis and the possibility that the sickest patients had already succumbed could influence study inclusion and outcome [4]. The exact aetiology of sepsis was not known, and so recurrence of the initial sepsis could not be excluded [4]. Moreover, this study is a retrospective analysis of a previous observational study (PICARD study) with all of the inherent pitfalls and limitations [10]. Mortality in septic patients with AKI was similar, irrespective of which came first, sepsis or AKI. The fact that so many patients with AKI develop sepsis and consequently, that AKI might be causing sepsis was barely addressed in any previous studies [4] and suggests the question: does AKI cause a state of immunoparalysis?

The phenomenon of immunoparalysis has been studied in more detail in the chronic kidney disease (CKD) population. A recent paper demonstrated that the prevalence of pneumonia was higher in CKD patients and especially in dialysis patients (including peritoneal home dialysis patients) suggesting thereby that CKD by itself is a state of reduced immunity and subsequently heightened risk for pneumonia and associated sepsis [11]. Another study showed that CKD patients and dialysis patients (including 
peritoneal home dialysis patients) were also at higher risk of developing flu-like infections despite influenza vaccination, suggesting decreased immunity in patients with $\mathrm{CKD}$ or, at least, decreased effectiveness of vaccination, as compared to controls [12]. Other studies demonstrated that hospitalized dialysed patients were more at risk for severe infection as compared to other hospitalized patients (and healthy control subjects), suggesting once again, that CKD patients (especially those requiring dialysis) are more prone to develop severe infections, probably because they have reduced immunity secondary to chronic uremia [13]. The propensity of patients with AKI to develop systemic inflammatory response syndrome (SIRS) and sepsis at least suggests that renal function, especially renal tubular cell dysfunction, plays a critical immunomodulatory role in individuals in stressed states [13, 14]. In vitro investigations clearly demonstrate proinflammatory activity of activated tubular cells as reflected by cytokine release [interleukin (IL)-6, IL-8] and leucocyte migration $[15,16]$.

There is evidence from recent human studies that inflammatory cascades initiated by endothelial dysfunction in SIRS are further dysregulated in the setting of AKI. Levels of the proinflammatory cytokines (such as IL-6) in plasma predict morbidity and even mortality in patients with AKI [17]. Furthermore, strategies that modulate the inflammatory response provide significant beneficial effects in experimental AKI [18]. Further elucidation of the underlying pathological processes in AKI will undoubtedly prove fruitful in preparing the way for modulation of these processes, thereby facilitating clinical interventions which could, in time, improve clinical outcome. Renal cell therapy and the development of new types of membranes designed to modify immunological dysregulation may indeed provide effective new approaches, though, at present, these remain firmly in the realm of speculation [14]. End stage renal disease (ESRD) is characterized by immune dysregulation presenting with clinical and biological immune deficiency despite chronic activation of immunocompetent cells, especially circulating neutrophils and monocytes [19]. This immune dysregulation develops in patients with chronic renal insufficiency prior to the initiation of chronic dialysis therapy, underlining the fact that, as in AKI, dialysis or other forms of renal replacement therapy are not the primer for immune dysregulation [21]. This finding of dysregulation of the immune state in chronic renal insufficiency may have profound implications for the AKI patient. In the hemodialyzed (HD) patient, the neutrophil dysfunction is borne out by the fact that infection is the second most common cause of death, approaching onequarter of the annual mortality rate in HD patients [14, 19]. This infection complication rate is not diminished with higher dialysis or hemofiltration dose or high-flux membrane utilization although clinical research in this field is ongoing [18]. Mortality due to sepsis occurs approximately more than 200 -fold more commonly in these patients compared to the general population $[14,20]$. Neither higher mortality rates due to infection nor higher rates of sepsis can be explained by infections secondary to vascular access alone $[19,20]$.

In addition, a chronic proinflammatory state exists in CKD patients, reflected by elevated biomarkers, specifically C-reactive protein and IL-6, as well as activation of circulating leukocytes, which correlates highly with accelerated atherosclerosis and early mortality [14]. The role, if any, of this latter finding in the AKI population is unclear, and extrapolation of findings from one population to the other must be approached with extreme caution [20].

Other recent studies have shown that AKI and its treatment can also aggravate an injury induced by an initial relatively localized infection. Nichol et al. [22] demonstrated in an animal model that buffering of hypercapnia as seen in lung-protective ventilation strategies, expedited by early bicarbonate infusion had significant drawbacks in AKI [22, 23]. Neutrophil gelatinase-associated lipocain (NGAL) is known as an antibacterial factor of natural immunity, is protective for the tubular cell, and is known to be an anti-oxidant factor [24]. NGAL is also known to play a regulatory role, somewhat like erythropoietin, in erythrocyte production [24]. A rapid reduction of NGAL during the course of AKI [particularly when renal replacement therapies (RRT) are applied] may dramatically increase the risk of subsequent infection and sepsis [24]. Erythropoietin (EPO) also exhibits some immunomodulatory effects [25] as demonstrated in a recent investigation whereby the addition of recombinant human erythropoietin normalized T lymphocyte activity as well as proliferative capacity [25]. EPO levels are reduced during AKI, thereby reducing the immune capacity of the body once AKI is established [25]. In some AKI patients, hyperparathyroidism has been described, though not as manifest as in CKD patients [26]. However, both parathyroid hormone (PTH) and teriparatide can inhibit the lymphocyte proliferation capacity [26], presenting us with another possible mechanism for immune modulation in AKI. Translating the knowledge of the myriad of possible mechanisms for immunoincompetence into viable novel therapeutic options that alter the acute inflammatory response in AKI and the chronic proinflammatory state of ESRD, however, remains a formidable challenge. While most of the medical approaches thus far, including antitumor necrosis factor-alpha therapy, thalidomide, and statins have been ineffective [27, 28], Humes et al. demonstrated that the use of renal cell therapy and cytopheretic membranes could lead to early clinical success and provide therapies resetting the dysregulated immunological states of AKI and CKD and thereby improving the clinical outcomes of these disease processes [14, 29].

In conclusion, Mehta et al. have demonstrated that sepsis after AKI is a problem which has been underestimated until now. The fact that mortality in septic patients 
with AKI is similar, irrespective of which came first, demonstrates this neatly.

Many potential mechanisms have been identified to explain the immunoparalysis occurring in early stage AKI prior to the initiation of any forms of RRT, which could predispose the AKI patient to sepsis. At the same time, these potential mechanisms could hold the key to novel therapeutic options that can have an impact on our daily clinical practice.

The authors rightly conclude that prospective studies are required to assess the derangements in host response that occur following AKI. Wise words, as the interplay between AKI and sepsis is far more complex than the simple question: which came first the chicken or the egg? After prospective studies, and only then, should a wellinformed clinical trial be designed, incorporating novel therapeutic options. The clinical consequences of this study however are obvious: every possible preventive measure has to be undertaken to prevent infections in patients suffering from AKI. This includes withholding invasive procedures whose clinical consequences are unclear.

\section{References}

1. Schrier RW, Wang W (2004) Acute renal failure and sepsis. N Engl J Med 351:159-169

2. Joannidis M, Metnitz PG (2005) Epidemiology and natural history of acute renal failure in the ICU. Crit Care Clin 21:239-249

3. Hoste EA, Lameire NH, Vanholder RC, Benoit DD, Decruyenaere JM, Colardyn FA (2003) Acute renal failure in patients with sepsis in a surgical ICU: predictive factors, incidence, comorbidity, and outcome. J Am Soc Nephrol 14:1022-1030

4. Levy EM, Viscoli CM, Horwitz RI (1996) The effects of acute renal failure on mortality. A cohort analysis. JAMA 275:1489-1494

5. Bagshaw SM, Uchino S, Bellomo R, Morimatsu H, Morgera S, Schetz M, Tan I, Bouman C, Macedo E, Gibney N, Tolwani A, van Oudemans-Straaten HM, Ronco C, Kellum JA (2007) Septic acute kidney injury in critically ill patients: clinical characteristics and outcomes. Clin J Am Soc Nephrol 2:431-439

6. Wan L, Bagshaw SM, Langenberg C, Saotome T, May C, Bellomo R (2008) Pathophysiology of septic acute kidney injury: what do we really know? Crit Care Med 36:198-203

7. Lerolle N, Nochy D, Guérot E, Bruneval P, Fagon J-Y, Diehl J-L, Hill G (2010) Histopathology of septic shock induced renal injury: apoptosis and leukocytic infiltration. Intensive Care Med 36:471-478

8. Joannes-Boyau O, Honoré PM, Boer W, Rose T (2010) Septic acute kidney injury and inflammatory apoptosis: never a lone ranger. Intensive Care Med 36:385-388

9. Mehta R, Bouchard J, Soroko S, Ikizler T, Paganini E, Chertow G, Himmelfarb J (2010) Sepsis as a cause and consequence of acute kidney injury: program to improve care in acute renal disease (PICARD). Intensive Care Med 36 doi:10.1007/s00134-010-2089-9
10. Mehta RL, Pascual MT, Soroko S, Savage BR, Himmelfarb J, Ikizier TA, Paganini EP, Chertow GM (2004) Spectrum of acute renal failure in the intensive care unit: the PICARD experience. Kidney Int 66:1643-1671

11. Coresh J (2009) CKD prognosis: beyond the traditional outcomes. Am J Kidney Dis 54:1-3

12. Cavdar C, Sayan M, Sifil A, Artuk C, Yilmaz N, Bahar H, Camsari T (2003) The comparison of antibody response to influenza vaccination in continuous ambulatory peritoneal dialysis, hemodialysis and renal transplantation patients. Scand J Urol Nephrol 37:71-76

13. Okusa MD (2002) The inflammatory cascade in acute ischemic renal failure. Nephron 90:133-138

14. Humes DH, Sobota JT, Ding F, Song JH (2010) A selective cytopheretic inhibitory device to treat the immunological dysregulation of acute and chronic renal failure. Blood Purif 29:183-190

15. Bijuklic K, Sturn DH, Jennings P, Kountchev J, Pfaller W, Wiedermann CJ, Patsch JR, Joannidis M (2006) Mechanisms of neutrophil transmigration across renal proximal tubular HK-2 cells. Cell Physiol Biochem 17:233-244

16. Bijuklic K, Jennings P, Kountchev J, Hasslacher J, Aydin S, Sturn D, Pfaller W, Patsch JR, Joannidis M (2007) Migration of leukocytes across an endothelium-epithelium bilayer as a model of renal interstitial inflammation. Am J Physiol Cell Physiol 293:C486C492

17. Liu KD, Altmann C, Smits G, Krawczeski CD, Edelstein CL, Devarajan P, Faubel S (2009) Serum interleukin-6 and interleukin- 8 are early biomarkers of acute kidney injury and predict prolonged mechanical ventilation in children undergoing cardiac surgery: a case-control study. Crit Care 13:R104
18. Anding K, Gross P, Rost JM, Allgaier D, Jacobs E (2003) The influence of uraemia and haemodialysis on neutrophil phagocytosis and antimicrobial killing. Nephrol Dial Transplant 18:2067-2073

19. Sherry B, Dai WW, Lesser ML, Trachtman H (2008) Dysregulated chemokine receptor expression and chemokine-mediated cell trafficking in paediatric patients with ESRD. Clin J Am Soc Nephrol 3:397-406

20. Sarnak MJ, Jaber BL (2000) Mortality caused by sepsis in patients with endstage renal disease compared with the general population. Kidney Int 58:1758-1764

21. Bellomo R, Palevsky PM, Bagshaw SM, Gibney N, McAlister FA, Honoré PM, Joannes-Boyau O, Prowle J, Haase M, Cruz DN, Ronco C (2010) Recent trials in critical care nephrology. Contrib Nephrol 165:299-309

22. Nichol AD, O'Cronin DF, Howell K, O'Brien S, Boylan J, O'Connor C, O'Toole D, Laffey JG, McLoughlin P (2009) Infection-induced lung injury is worsened after renal buffering of hypercapnic acidosis. Crit Care Med 37:2953-2961

23. Honoré PM, Joannes-Boyau O, Boer W, Janvier G (2008) CRRT induced alkalosis in ICU patients: the questions start here. Crit Care Med 36:1665-1666

24. Bolignano D, Coppolino G, Donato V, Lacquaniti A, Bono C, Buemi M (2010) Neutrophil gelatinase-associated lipocain (NGAL): a new piece of the anaemia puzzle? Med Sci Monit 16:131-135

25. Lisowska KA, Debska-Slizien A, Radzka M, Witkowski JM, Rutkowski B, Bryl E (2010) Recombinant human erythropoietin treatment of chronic renal failure patients normalizes altered phenotype and proliferation of $\mathrm{C}$ positive $\mathrm{T}$ lymphocytes. Artif Organs 34:77-84 
26. Castellanos M, Jung E, Park SY, Schuller-Levis G, Odaimi M, Elsayegh S, Kleiner M, Elsoueidi R, Shtaynberg N, Park E (2010) Effect of parathyroid hormone and teriparatide on immune adherent and non-adherent leukocytes. Clin Nephrol 74:83-90
27. Vielhauer V, Mayadas TN (2007) Functions of TNF and its receptors in renal disease: distinct roles in inflammatory tissue injury and immune regulation. Semin Nephrol 27:286-308

28. Gluba A, Rysz J, Banach M (2010) Statins in patients with chronic kidney disease: why, who and when? Expert Opin Pharmacother 11:2665-2674
29. Humes HD, Weitzel WF, Bartlett RH, Swaniker FC, Paganini EP, Luderer JR, Sobota J (2004) Initial clinical results of the bioartificial kidney containing human cells in ICU patients with acute renal failure. Kidney Int 66:1578-1588 